\title{
Boundary Layers in Cataclysmic Variables and Pre-Main-Sequence Stars
}

\author{
Robert Popham \\ Harvard-Smithsonian Center for Astrophysics, MS 51, 60 Garden St., \\ Cambridge, MA 02138
}

\begin{abstract}
The boundary layer region, where the accretion disk meets the accreting star, is crucial to understanding the spectra and evolution of accretion disk systems. Recent numerical modeling of the flow of the accreting material and radiation in the boundary layer has provided a better understanding of this region. I will describe the "standard" boundary layer structure predicted by the models for the case where the boundary layer is optically thick and geometrically thin in the vertical direction. Large variations in this structure can occur when the boundary layer becomes optically thin or geometrically thick. Boundary layer models have been applied to cataclysmic variables and accreting pre-main-sequence stars. I will discuss the boundary layer spectra predicted by the models and how well they agree with observations of these objects. The boundary layer also controls the transfer of angular momentum and energy between the disk and star. This can have important effects upon pre-main-sequence stellar evolution.
\end{abstract}

\section{Introduction}

The boundary layer (BL) is the transition region between an accretion disk and the accreting star. The name "boundary layer" implies that this region contains a sharp transition between the rapidly rotating disk and the slowly rotating star. While this is true in some systems, in others the transition from disk to star may be more gradual, or the star may be rotating rapidly. I will therefore use the term "boundary layer" in a more general way, to simply mean the transition region between disk and star.

The importance of the boundary layer region is clear when considering the energy balance of the accretion process. If the accretion disk rotates at the Keplerian velocity near the stellar surface, the accreting material still retains half of the gravitational energy released by accretion in the form of rotational kinetic energy: $(1 / 2) \dot{M} v_{K}^{2}\left(R_{*}\right)=(1 / 2) G M_{*} \dot{M} / R_{*}$.

The fate of the remaining energy is decided within the boundary layer region. Most of the energy may be radiated away, in which case the boundary layer will produce a luminosity comparable to that from the disk. Thus the BL will make an important contribution to the spectrum of the accreting system; since the BL is smaller than the disk, it will dominate the high-energy end of the spectrum. 
The remainder of the energy released by accretion will go into spinning up the star, or into heating the accreting material which subsequently falls onto the star. Thus the BL mediates the transfer of angular momentum and energy between disk and star.

\section{Modifying the Standard Disk Equations to Treat the Boundary Layer}

The standard disk equations (Shakura \& Sunyaev 1973) cannot treat the BL, and were not designed to do so. They make a number of assumptions which, while valid in a thin disk, will not apply to the transition region from the disk to the star.

\subsection{Radial Momentum Balance}

First we consider the radial momentum balance equation. The thin disk equations simply assume $\Omega=\Omega_{K}$, so that gravity is balanced exactly by centrifugal acceleration. Since by definition $\Omega$ must reach the stellar rotation rate $\Omega_{*}$, this cannot be true in the BL.

In the star, gravity is balanced by the radial pressure gradient. Thus in the $\mathrm{BL}$, we expect that the accreting material must make the transition from rotational to pressure support. If the radial forces acting upon the accreting material are not exactly balanced, it will undergo some radial acceleration. Including the pressure gradient and radial acceleration terms in the radial momentum balance equation gives

$$
\left(\Omega^{2}-\Omega_{K}^{2}\right) R=\frac{1}{\rho} \frac{d P}{d R}+v_{R} \frac{d v_{R}}{d R} .
$$

\subsection{Angular Momentum Balance}

The angular momentum balance in the boundary layer and disk is determined by two terms. The first is the angular momentum carried inward by the accreting material. The second is the angular momentum transferred down the $\Omega$ gradient by viscosity. The angular momentum accretion rate $\dot{J}$ is the sum of the two terms; in a steady state, it must be constant with radius.

$$
\dot{J}=\dot{M} \Omega R^{2}-\dot{M}\left(\frac{\nu}{v_{R}} \frac{d \Omega}{d R}\right) R^{2}
$$

The viscous term carries angular momentum outward in regions where $\Omega$ decreases with decreasing radius $(d \Omega / d R>0)$, as in the disk, and it carries angular momentum inward where $d \Omega / d R<0$, as is often true in the boundary layer. If $\Omega$ reaches a maximum between the disk and boundary layer, the viscous term will be zero there, since $d \Omega / d R=0$. At this point, we simply have $\dot{J}=$ $\dot{M} \Omega R^{2}$, and since $\dot{J}$ is constant with radius, this sets the value of $\dot{J}$ for the entire disk.

In the standard thin disk model, we assume that $\Omega=\Omega_{K}$, and that $\Omega$ reaches a maximum value $\Omega \simeq \Omega_{K}\left(R_{*}\right)$ very close to the stellar surface at $R \simeq R_{*}$. These assumptions give an angular momentum accretion rate 
$\dot{J}=\dot{M} \Omega_{K}\left(R_{*}\right) R_{*}^{2}$, and by substituting this value for $\dot{J}$ in the expression given above, one finds

$$
\nu \Sigma=\frac{\dot{M}}{3 \pi}\left[1-\left(\frac{R_{*}}{R}\right)^{1 / 2}\right]
$$

This expression is then used in obtaining the standard set of equations for the radial structure of a thin disk. Clearly, it breaks down at $R=R_{*}$, as do all of the thin disk equations which depend on it.

To study the BL, we need to avoid these assumptions, and so we use the more general version of the angular momentum equation given above.

\subsection{Energy Balance}

The standard thin disk model assumes a local balance between the viscous dissipation of energy and the energy radiated away from the disk surface at any given radius:

$$
\frac{1}{2} \nu \Sigma\left(R \frac{d \Omega}{d R}\right)^{2}=\frac{3 G M_{*} \dot{M}}{8 \pi R^{3}}\left[1-\left(\frac{R_{*}}{R}\right)^{1 / 2}\right]=F_{V} .
$$

In the $\mathrm{BL}$, there are several reasons why this local balance may not be maintained. The very concentrated energy dissipation within the BL means that radial gradients will become large, and energy will be transported to other nearby radii. Some energy may be advected inward with the accreting material, and radiative flux may transfer energy down the local radial temperature gradient. When these radial energy transport terms are included, the energy equation becomes:

$$
\frac{1}{2} \nu \Sigma\left(R \frac{d \Omega}{d R}\right)^{2}-F_{V}=v_{R} \Sigma T \frac{d S}{d R}+\frac{1}{R} \frac{d}{d R}\left(R H F_{R}\right)
$$

\subsection{Solution Methods}

The resulting set of disk equations are basically the slim disk equations (Paczyński \& Bisnovatyi-Kogan 1981; Muchotrzeb \& Paczyński 1982), which were originally developed to treat transonic flow problems in disks around black holes. Unlike the standard thin disk equations, which can be solved analytically, these are 1-D, steady-state differential equations contain radial derivatives which contain radial derivatives. The equations tend to be rather stiff, but can be solved using relaxation methods. Alternatively, Regev and collaborators have used a matched asymptotic expansion method to solve for the boundary layer structure. The equations can also be solved in time-dependent form, as Godon and collaborators have done using spectral methods. Finally, two-dimensional, time-dependent models which compute the boundary layer structure in both the radial and vertical directions are starting to become feasible, and have been constructed by Kley and collaborators and by Hujeirat. 

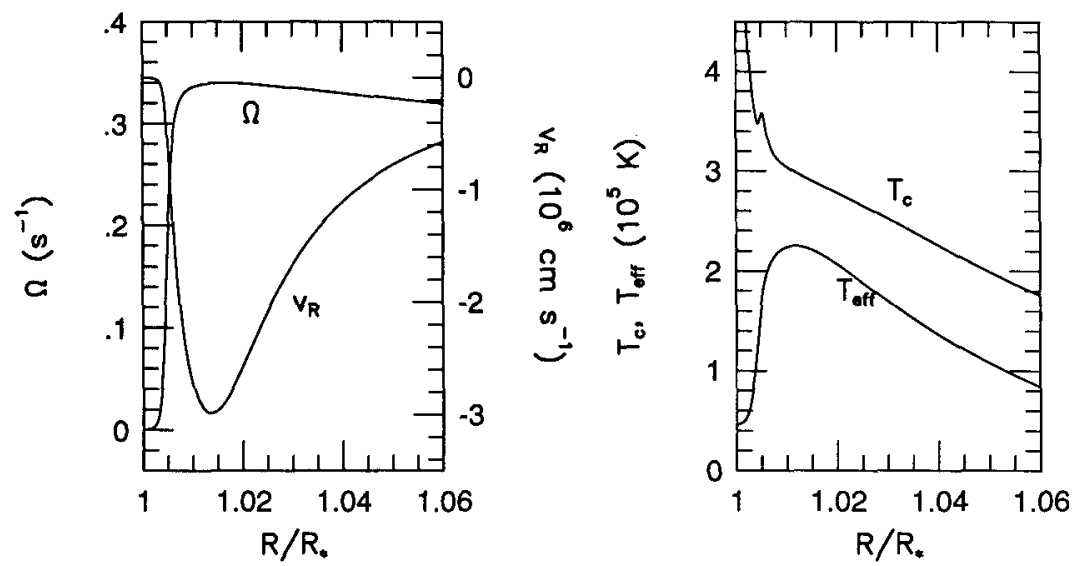

Figure 1. A boundary layer solution for a high- $\dot{M} \mathrm{CV}$ with $\dot{M}=$ $10^{-8} M_{\odot} \mathrm{yr}^{-1}$ and a white dwarf mass of $0.6 M_{\odot}$ and radius of $8.7 \times$ $10^{8} \mathrm{~cm}$. The left panel shows the angular and radial velocities $\Omega$ and $v_{R}$, and the right panel shows the central and effective temperatures $T_{c}$ and $T_{\text {eff }}$.

\section{Application to Cataclysmic Variables and Pre-Main-Sequence Stars}

\subsection{Cataclysmic Variables}

High- $\dot{M}$ systems (Popham \& Narayan 1995; Godon et al. 1995; Hujeirat 1995; Kley 1989,1991$)$

By solving the slim disk equations described above, we can find solutions for a wide range of different accretion disk systems. For high- $\dot{M}$ cataclysmic variables (CVs), we find that the $B L$ is optically thick and geometrically thin in the vertical direction. As a result, the BL has a "standard" structure consisting of a narrow "dynamical boundary layer" where $\Omega$ drops from $\sim \Omega_{K}$ to $\Omega_{*}$, and a wider "thermal boundary layer" where the BL luminosity which has diffused radially outward into the inner disk is radiated from the disk surface. The radial width of the dynamical $\mathrm{BL}$ is generally quite small, a few times $H^{2} / R$, or less than $1 \%$ of the stellar radius, while the thermal BL extends over a width of a few times the disk height $H$, or $\sim 10 \%$ of a stellar radius. The size of the thermal BL determines the area over which the BL luminosity must be radiated, and thus sets the effective temperature of the BL. For high- $\dot{M} \mathrm{CVs}$ $\left(\dot{M}=10^{-8.2}-10^{-7} M_{\odot} \mathrm{yr}^{-1}\right)$, we find peak values of $T_{\text {eff }} \simeq 2-3.5 \times 10^{5} \mathrm{~K}$.

\section{Low- $\dot{M}$ systems (Narayan \& Popham 1993)}

In CVs with lower values of $\dot{M}$, the BL becomes optically thin as the density drops so that the accreting material cannot cool efficiently, and its temperature rises. As a result, a large part of the BL becomes very hot and reaches a very low density. The energy dissipated within this hot region heats the accreting gas and is advected across the hot region with the gas. When it approaches 

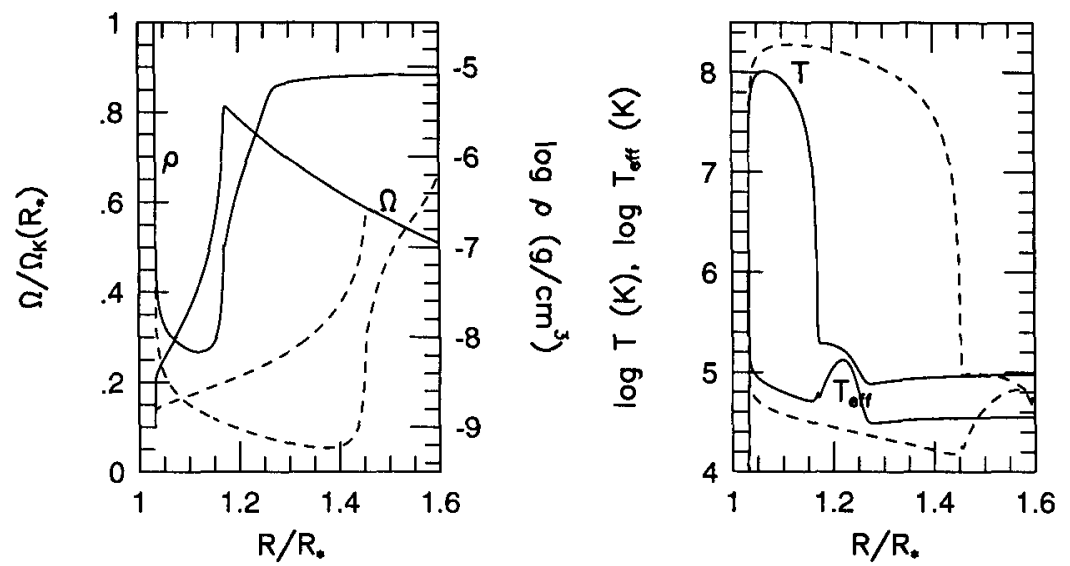

Figure 2. Boundary layer solutions for low- $\dot{M}$ CVs with $\dot{M}=$ $10^{-9.5} M_{\odot} \mathrm{yr}^{-1}$ (solid line) and $\dot{M}=10^{-10.5} M_{\odot} \mathrm{yr}^{-1}$ (dashed line) and a white dwarf mass $1.0 M_{\odot}$ and radius $5 \times 10^{8} \mathrm{~cm}$. The left panel shows the angular velocity $\Omega$ and the density $\rho$, and the right panel shows the central and effective temperatures $T$ and $T_{\text {eff }}$.

the surface of the star, this gas becomes optically thick again, and radiates the stored energy outward across the hot region, where it heats the optically thick inner disk. Thus both the advective and radiative radial energy transport terms in the energy equation play important roles in optically thin boundary layers. The hot region in these systems reaches very high temperatures $T \simeq 10^{8} \mathrm{~K}$, and therefore produces hard X-rays.

Comparison with Observations The transition from an optically thick to an optically thin BL with decreasing $\dot{M}$ results in a corresponding transition from EUV and soft X-ray emission from high- $\dot{M}$ CVs to hard X-rays from low- $\dot{M}$ CVs. This agrees with the trend seen in a large sample of observed CVs by Patterson \& Raymond (1985) and in individual dwarf novae as they go between outbursts and quiescent states (Jones \& Watson 1992; Wheatley et al. 1996).

The general expectation for systems where the accreting star is rotating slowly is that the boundary layer luminosity should be comparable to that of the disk; each should be about half of the total accretion luminosity. In optically thick CVs, the bulk of the BL luminosity is in the EUV, where it is difficult to observe. Among the three systems where EUV observations have been feasible, the EUV fluxes are comparable to UV fluxes from disk only in U Gem (Long et al. 1996), but are much smaller than the disk fluxes in SS Cyg and VW Hyi (Mauche et al. 1995, Mauche 1996).

\subsection{Pre-Main-Sequence Disks}

T Tauri Stars (Popham et al. 1993; Lioure \& Le Contel 1994; Regev \& Bertout 1995; Godon 1996a,b; Kley \& Lin 1996) 

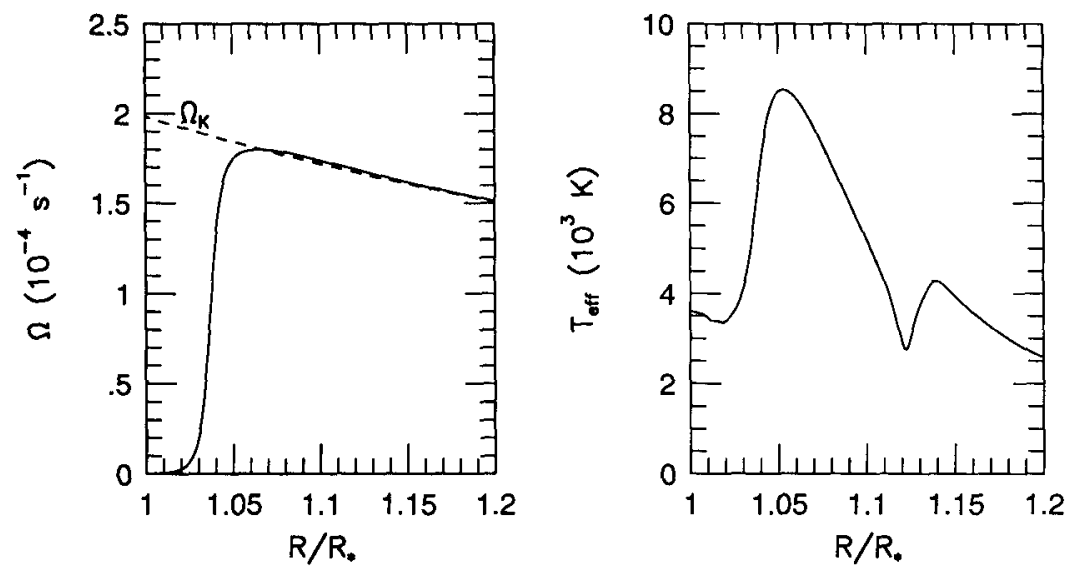

Figure 3. A boundary layer solution for a T Tauri star with $\dot{M}=$ $10^{-7} M_{\odot} \mathrm{yr}^{-1}$ and a stellar mass $1 M_{\odot}$ and radius $2.23 R_{\odot}$. The left panel shows the angular velocity $\Omega$, with $\Omega_{K}$ indicated by a dashed line, and the right panel shows the effective temperature $T_{e f f}$.

In T Tauri stars, as in high- $\dot{M}$ CVs, the BL is optically thick but geometrically thin in the vertical direction. As a result, the BL has the standard structure, with a narrow dynamical $\mathrm{BL}$ and a wider thermal $\mathrm{BL}$. The radial width of the thermal BL is a few percent of $R_{*}$, and it reaches a peak $T_{\text {eff }} \simeq 8500 \mathrm{~K}$ for an accretion rate $\dot{M}=10^{-7} M_{\odot} \mathrm{yr}^{-1}$ onto a $1 M_{\odot}$ star. An emitting region with this size and temperature can provide the blue excess continuum emission or "veiling" in T Tauri spectra (Basri \& Bertout 1989; Hartigan et al. 1991).

Other authors (Lioure \& Le Contel 1994; Regev \& Bertout 1995) use a boundary condition on the temperature at the star-disk interface which causes most of the BL luminosity to be radiated inward into the star rather than outward into the inner disk. This reduces the effective temperature of the BL region, producing a so-called "cool" BL. However, since this inwardly-directed luminosity will rapidly heat up the outer layer of the star, it seems that it would be difficult to maintain the temperature dictated by the boundary condition.

FU Orionis Objects (Popham et al. 1993; Popham 1996; Popham et al. 1996; Godon 1996b; Kley \& Lin 1996)

FU Orionis objects are believed to be $\mathrm{T}$ Tauri stars in which the disk undergoes outbursts with very high values of $\dot{M} \simeq 10^{-4} M_{\odot} \mathrm{yr}^{-1}$. As a result of these high accretion rates, the disk is thick: $H / R \sim 0.3-0.5$. This produces a much wider BL - both the dynamical and thermal BLs have widths comparable to $R_{*}$. Since the BL luminosity is radiated from a large region, it has a rather low effective temperature. The peak values of $T_{\text {eff }} \simeq 7000 \mathrm{~K}$ are comparable to those of the inner disk, so no distinct BL component is visible in the spectrum. This agrees with observations of these systems, which show very little UV flux. 

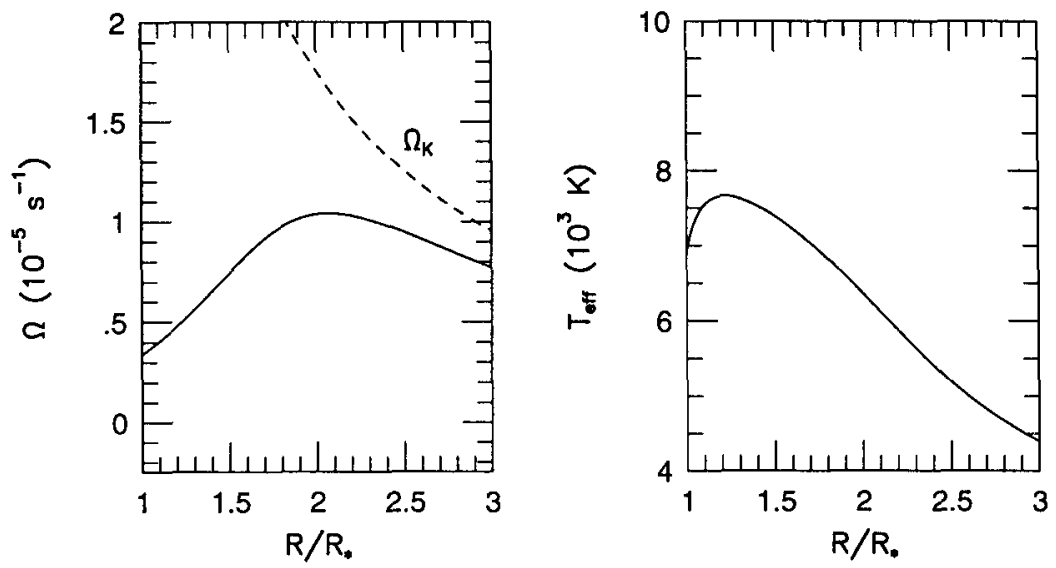

Figure 4. A boundary layer solution for an FU Orionis object with $\dot{M}=10^{-4.15} M_{\odot} \mathrm{yr}^{-1}$ and a stellar mass $0.5 M_{\odot}$ and radius $3 \times 10^{11} \mathrm{~cm}$. The left panel shows the angular velocity $\Omega$, with $\Omega_{K}$ indicated by a dashed line, and the right panel shows the effective temperature $T_{\text {eff }}$.

Angular Momentum Accretion in FU Orionis Objects (Popham 1996; Popham et al. 1996)

Because the accretion rates in FU Orionis objects are so high, accretion can have significant effects on the underlying star; for example, angular momentum accretion during outbursts can affect the spin evolution of the star.

In the standard picture of angular momentum accretion from a disk, the angular momentum accretion rate $\dot{J}$ is set by the existence of a maximum in $\Omega$ close to $R_{*}$ (as discussed earlier). If $\Omega \simeq \Omega_{K}$ in the disk, the resulting angular momentum accretion rate will be $\dot{J} \simeq \dot{M} \Omega_{K}\left(R_{*}\right) R_{*}^{2}$.

Once the accreting star spins up enough, there will no longer be a maximum in $\Omega$. Instead $d \Omega / d R<0$ everywhere, so viscosity will transport angular momentum outwards from the star into the disk. At this point, there is no longer any constraint on $\dot{J}$. Thus, as the star spins up, $\dot{J}$ drops until it reaches $\simeq 0$ at an equilibrium stellar rotation rate $\Omega_{*}=\Omega_{e q}$. Since it is not accreting any more angular momentum, the star should continue to rotate at $\Omega_{e q}$. If the star were to continue to spin up to $\Omega>\Omega_{e q}, \dot{J}$ would become negative, so that the disk would remove angular momentum from the star and spin it back down (Popham \& Narayan 1991; Paczyński 1991).

The maximum value of $\Omega$ in a thin disk is close to $\Omega_{K}\left(R_{*}\right)$. Thus, the star must spin up very close to breakup before reaching $\Omega_{e q} \simeq \Omega_{K}\left(R_{*}\right)$. On the other hand, for a thick disk, as in FU Orionis systems, the boundary layer is wide, so that the maximum in $\Omega$ occurs far from $R_{*}$. Also, radial pressure support is important in thick disks, so that $\Omega$ tends to be substantially sub-Keplerian. The result is that the maximum value of $\Omega$ in these disks is well below $\Omega_{K}\left(R_{*}\right)$. Therefore, the star should stop spinning up when it reaches $\Omega_{e q} \ll \Omega_{K}\left(R_{*}\right)$. 

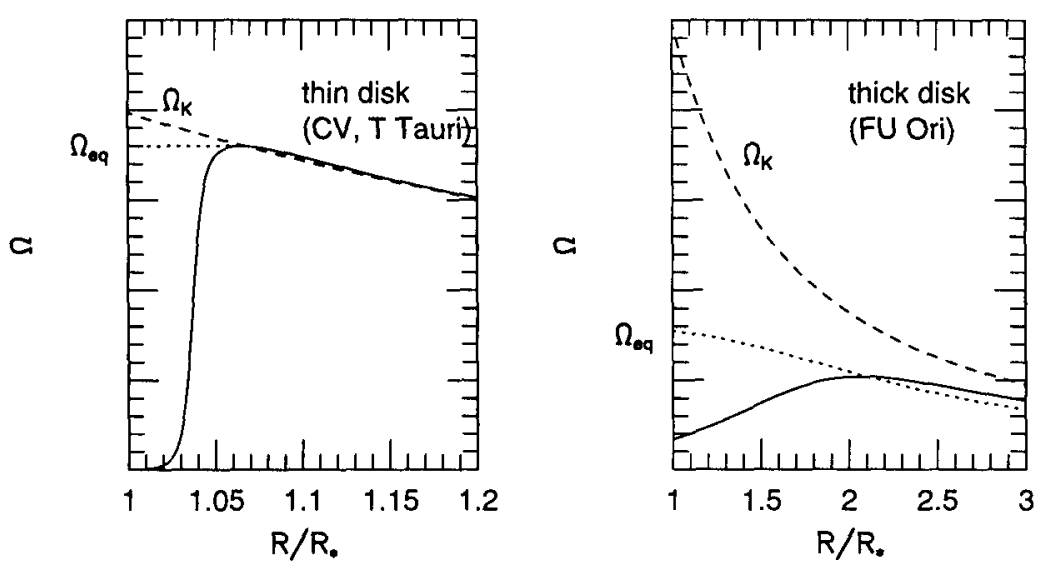

Figure 5. The left panel shows a typical $\Omega$ profile for a thin disk system (solid line); in order to reach the equilibrium stellar rotation rate $\Omega_{e q}$ (dotted line), the star must spin up to close to the breakup rotation rate $\Omega_{K}\left(R_{*}\right)$. The right panel shows similar profiles for a thick disk FU Orionis system; here $\Omega_{e q}$ is well below the breakup value.

FU Orionis outbursts will cause the underlying T Tauri stars to spin up or down, depending on the star's rotation rate prior to the outburst, toward $\Omega_{e q}$. Current estimates suggest that the mass accreted during repeated outbursts is comparable to that accreted during the $\mathrm{T}$ Tauri phases; the same should hold true for angular momentum accretion. If so, then the outbursts will keep $T$ Tauri star rotation rates close to $\Omega_{e q}$.

$\mathrm{T}$ Tauri stars are observed to rotate quite slowly, a fact which is generally attributed to magnetic accretion processes. However, the equilibrium rotation rates $\Omega_{e q}$ during $\mathrm{FU}$ Orionis outbursts are comparable to the observed rotation rates of $\mathrm{T}$ Tauri stars, suggesting that the outbursts could be responsible for the slow rotation rates. It is important to note, though, that the radii of the accreting stars in FU Orionis disk models tend to be a factor of 2-3 larger than the radii of T Tauri stars, as discussed further below.

The observed spectra and line profiles of FU Orionis objects provide information on the run of velocity and temperature in the disk. By calculating spectra and line profiles from our solutions, we have found that solutions with fairly conventional BLs do not match the observations well. The boundary layer region tends to be too hot, and rotates too slowly, producing centrally-peaked lines. On the other hand, $\dot{J}=0$ equilibrium solutions produce lower BL temperatures and double-peaked line profiles which agree with the observed spectra and line profiles of FU Orionis and V1057 Cygni.

\section{Energy Advection in FU Orionis Objects (Popham et al. 1996)}

Rapid accretion during FU Orionis outbursts also carries large amounts of energy into the accreting star. Boundary layer solutions for FU Orionis objects demonstrate that the BL and disk are highly optically thick in the vertical 

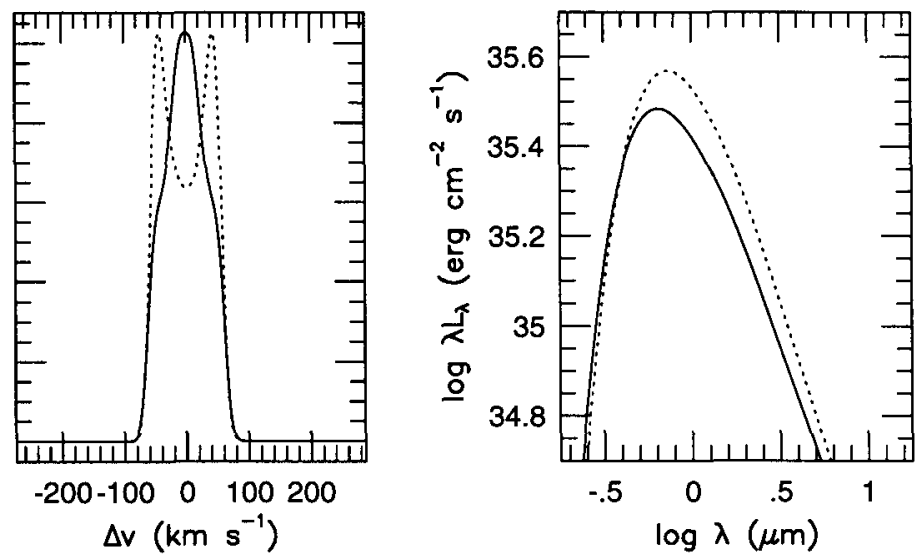

Figure 6. Line profiles (left panel) and spectra (right panel) for two boundary layer solutions for an FU Orionis star which have the $\Omega$ profiles shown in the right panel of Fig. 5: a high- $\dot{J}$ solution (solid line) and a $\dot{J}=0$ solution (dotted line). Both have $\dot{M}=10^{-4.15} M_{\odot} \mathrm{yr}^{-1}$, $M_{*}=0.5 M_{\odot}$ and $R_{*}=3 \times 10^{11} \mathrm{~cm}$. The $\dot{J}=0$ solution produces a double-peaked line profile and a redder, more luminous spectrum, in agreement with observations.

direction, so that the accreting material cannot cool efficiently. Therefore, the disk is geometrically thick, indicating that the energy content of the accreting material is a substantial fraction of gravitational energy released by accretion: $(H / R)^{2}=\left(c_{s} / \Omega_{K} R\right)^{2}=c_{s}^{2} /(G M / R)=\dot{M} c_{s}^{2} / L_{a c c}$.

The energy stored in the accreting material when it reaches the stellar surface is advected into the star. For the FU Orionis solutions shown above, we find that the rate of energy advection into the star is $\simeq 0.375 L_{a c c}>100 L_{\odot}$. This very rapid deposition of energy would probably cause the star's envelope to expand rapidly. All disk models for FU Orionis objects appear to require stellar radii 2-3 times larger than those of $T$ Tauri stars, and so perhaps this apparent expansion during outbursts is due to the effects of energy advection.

Acknowledgments. This work was supported by grants NASA NAG52837 and NSF AST-9423209.

\section{References}

Basri, G., \& Bertout, C. 1989, ApJ, 341, 340.

Godon, P. 1996a, ApJ, 463, 674.

Godon, P. 1996b, MNRAS, 279, 1071.

Godon, P., Regev, O., Shaviv, G. 1995, MNRAS, 275, 1093.

Hartigan, P., et al. 1991, ApJ, 382, 617. 
Hujeirat, A. 1995, A\&A, 295, 268.

Jones, M. H., \& Watson, M. G. 1992, MNRAS, 257, 633.

Kley, W. 1989, A\&A, 222, 141.

Kley, W. 1991, A\&A, 247, 95.

Kley, W., \& Lin, D. N. C. 1996, ApJ, 461, 933.

Lioure, A., \& Le Contel, O. 1994, A\&A, 285, 185.

Long, K. S., Mauche, C. W., Raymond, J. C., Szkody, P., Mattei, J. A. 1996, ApJ, 469, 841.

Mauche, C. W., Raymond, J. C., Mattei, J. A. 1993, ApJ, 446, 842.

Mauche, C. W. 1996, in Cataclysmic Variables and Related Objects, eds. A. Evans, J. H. Wood (Dordrecht: Kluwer).

Muchotrzeb, B., \& Paczyński, B. 1982, Acta Astron., 32, 1.

Narayan, R., \& Popham, R. 1993, Nature, 362, 820.

Paczyński, B. 1991, ApJ, 370, 597.

Paczyński, B., \& Bisnovatyi-Kogan, B. 1981, Acta Astron., 31, 283.

Patterson, J. \& Raymond, J. C. 1985, ApJ, 292, 535.

Popham, R. 1996, ApJ, 467, 749.

Popham, R., Kenyon, S., Hartmann, L., \& Narayan, R. 1996, ApJ, in press.

Popham, R., \& Narayan, R. 1991, ApJ, 370, 604.

Popham, R., \& Narayan, R. 1995, ApJ, 442, 337.

Popham, R., Narayan, R., Hartmann, L., \& Kenyon, S. 1993, ApJ, 415, L127.

Regev, O., \& Bertout, C. 1995, MNRAS, 272, 71.

Shakura, N. I., \& Sunyaev, R. A. 1973, A\&A, 24, 337.

Wheatley, P. J., et al. 1996, A\&A, 307, 137.

\section{Discussion}

N. Arav: What is the nature of the viscosity used in your calculations and is radiative viscosity important in the appropriate phase space?

R. Popham: We use an alpha-prescription which has been modified to use the radial pressure scale height rather than the vertical one in regions where the radial is the smaller of the two. I don't think radiative viscosity is important in any of these systems.

K. Beuermann: Do you assume instantaneous dissipation of the kinetic energy in the boundary layer or do you allow also for long term storage of energy in form of a rotating belt?

R. Popham: Some of the solutions correspond to rotating stars, so in these some of the kinetic energy is retained. We also allow for storage of some of the dissipated energy in the form of heating of the accreting gas, which can be important in the advecting systems.

D. Meier: I want to follow up on the previous question. How do you treat the viscous interaction between the disk and the star? It would seem that this is a 
complex problem - at least as difficult as the viscosity problem in the disk itself. Do you simply propagate the $\alpha$-model viscosity from the disk into the star and use the same values of the parameters?

R. Popham: Yes, we use the same set of equations and the same viscosity throughout the disk, boundary layer, and the outer layers of the star. This is the simplest assumption to make, but I agree that it would be very interesting to see what happens when this assumption is relaxed.

$R$. Wehrse: Can you estimate the uncertainty in the effective temperatures you gave due to uncertainties in the opacities (lines?) and due to the fact that no detailed radiative transfer is taken into account?

R. Popham: I think the effective temperatures should be reasonably accurate, but I can't give you a quantitative estimate of the uncertainties due to these effects. In the pre-main-sequence disks, where the temperature dependence of the opacity is more complex, we have used tabulated opacities, but none of the calculations include any line cooling. 